\title{
Shape from Focus with Adaptive Focus Measure and High Order Derivatives
}

\author{
Yuval Frommer ${ }^{1}$ \\ yuvalfrommer@yahoo.com \\ Rami Ben-Ari² \\ ramib@il.ibm.com \\ Nahum Kiryati ${ }^{1}$ \\ nahum.kiryati@gmail.com
}

\author{
${ }^{1}$ School of Electrical Engineering \\ Tel-Aviv University \\ Tel-Aviv, Israel \\ ${ }^{2}$ IBM Research \\ Haifa, Israel
}

Shape From Focus (SFF) methods frequently use a single focus measure to obtain a depth map. Common focus measures are fixed and spatially invariant. In this paper we present a framework to create an adaptive focus measure based on ensemble of basis focus operators defined by the image derivatives in various orders and directions. The method adaptively chooses the most informative combination of derivatives at each pixel by weighting the contribution of each derivative accordingly. Weighting is achieved using the curve standard deviation (CSTD) which measures how peaked the distribution of focus measures is. The CTSD characteristic is used both during focus measure calculation and aggregation. This approach effectively generalizes some of the existing measures e.g. ML [2]. We assess the performance of our new approach by extensive experiments showing a significant increase in accuracy compared to state-of-the-art focus measures and with comparison to other methods which perform aggregation e.g. [1] and more.

Agglomeration of the focus measures in an image stack $I_{z}(x, y)$ builds up the focus volume $\phi:(X \times Y) \times Z \mapsto \mathbb{R}$, assigning a focus measure to each point in the image stack. We now define a linear space with the basis functions as the image derivatives, in different orders and directions:

$$
\phi_{i j}=\left|\frac{\partial^{i} I}{\partial \mathbf{x}_{j}^{i}}\right|
$$

where $i, j$ index the the derivative order and the discrete directions respectively. This paper suggests a focus measure based on a linear combination of image derivatives represented by a vector in the focus-measure space:

$$
\psi(x, y, z)=\sum_{i=1}^{n} \sum_{j=1}^{m} \alpha_{i j}(x, y) \hat{\phi}_{i j}(x, y, z)
$$

where, $\alpha_{i j}$ denote the coefficients of the basis focus components. Note that existing image based derivative focus measures e.g. ML [2] become a particular case in this framework. We call the new focus measure $\psi$ as the Adaptive High Order (AHO) focus measure. A focus curve should ideally be a symmetric uni-modal function with a narrow peak. To this end, we introduce a reliability measure for a given focus curve as curves' standard deviation (CSTD). The coefficients $\alpha_{i j}(x, y)$ are then set locally according to CSTD.

Once the focus volume in (2) is computed, a standard procedure includes an aggregation process. Using the newly introduced CSTD we suggest a novel iterative aggregation scheme:

$$
\tilde{\psi}_{t}(x, y, z)=\sum_{\left(x^{\prime}, y^{\prime}\right) \in \mathrm{W}} \hat{w}\left(x^{\prime}, y^{\prime}\right) \psi_{t-1}\left(x+x^{\prime}, y+y^{\prime}, z\right)
$$

where $W$ denotes the aggregation window and $\hat{w}$ represents the associated weights set according to CSTD measure. Finally the depth at each point is computed as the focus curve centroid introducing a non-parametric cue for depth estimation.

To evaluate the new focus measure we conduct a quantitative comparison in terms of depth map RMSE (Root Mean Square Error) and report the results in Table 1. Notably the AHO achieves results that are more

\begin{tabular}{|lcccc|}
\hline Object & GLV & ML [2] & WAV [3] & Ours \\
\hline \hline Cloth & 2.6 & 1.42 & 1.78 & $\mathbf{0 . 7 6}$ \\
Synthetic-Cone & 7.05 & 3.73 & 1.05 & $\mathbf{0 . 3 3}$ \\
Middlebury-Cones & 3.42 & 1.56 & 2.49 & $\mathbf{1 . 1 7}$ \\
Cube & 5.18 & 3.63 & 1.67 & $\mathbf{0 . 7 6}$ \\
\hline Average & 4.56 & 2.59 & 1.75 & $\mathbf{0 . 7 6}$ \\
\hline
\end{tabular}

Table 1: Comparison of focus measure operators in terms of depth map RMSE. Best results are in bold.

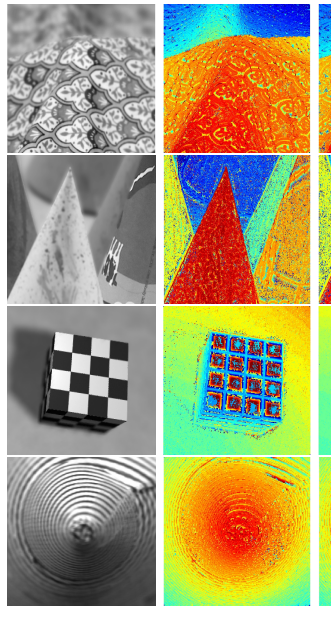

GLV

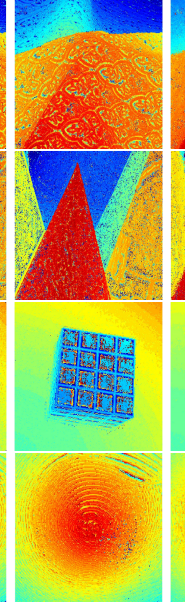

SML [2]

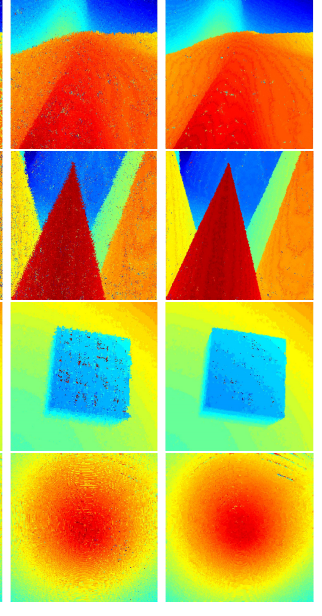

WAV [3]
Ours
Figure 1: Comparison of focus measures. Note the significant improvement obtained using the proposed focus measure.

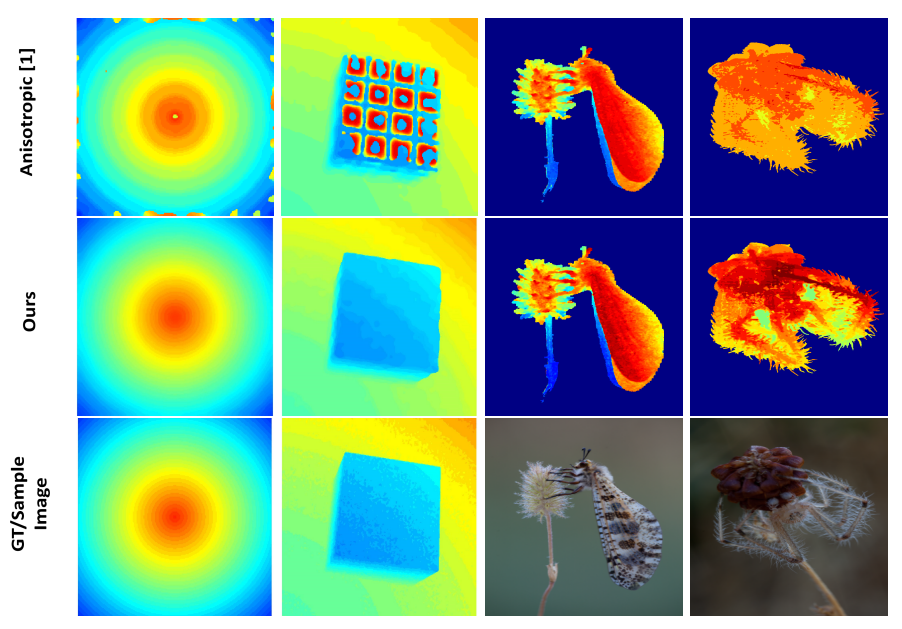

Figure 2: Comparison with a full pipeline method.

presents a qualitative comparison with the state-of-the-art focus measures in the literature. The results show the significant improvement obtained by our AHO focus measure, well coping with different textures as well as intensity and depth edges. Next, we present in Fig. 2 the performance of our complete pipeline for shape reconstruction using the proposed focus measure, aggregation and reconstruction approach. Note that the Antlion and the Spider depth maps at the third and fourth columns are driven from image stacks captured in the wild with a SLR camera.

[1] M. T. Mahmood, S. O. Shim, and T. S. Choi. Depth and image focus enhancement for digital cameras. International Symposium on Consumer Electronics, pages 50-53, 2011.

[2] S. K. Nayar and Y. Nakagawa. Shape from focus. TPAMI, 16(8): 824-831, 1994.

[3] G. Yang and B. J. Nelson. Wavelet-based autofocusing and unsupervised segmentation of microscopic images. In Int. Conference on Intelligent Robots and Systems, volume 3, pages 2143-2148, 2003. 